\title{
INDICATOR DILUTION CURVES IN MITRAL VALVULAR DISEASE
}

\author{
BY \\ P. G. F. NIXON AND H. M. SNOW \\ From the Departments of Thoracic Surgery and Medicine in the General Infirmary at Leeds \\ Received January 18, 1962
}

Mitral regurgitation takes place through orifices that are small and severely obstructed or through orifices that are large and seriously incompetent. In disabled patients with atrial fibrillation the predominant lesion can be diagnosed from the diastolic murmur and third heart sound (Nixon and Wooler, 1960; Nixon, 1961a), and from the left atrial stasis wave (Nixon and Wooler, 1961). Measurement of regurgitation would permit the ranking of patients with a particular lesion and simplify the assessment of surgical treatment. The indicator-dilution curve is the method of measurement most likely to succeed, but interpretation is difficult because distortion results from abnormalities of blood flow and heart size as well as from regurgitation. Simple methods of recording and analysing dye curves have a useful place in the assessment of patients with mitral valvular disease, but reference to published reports tends to obscure this fact because some methods of recording and analysis are prohibitively complicated, and because it may be difficult to decide how closely applicable to disease states are theoretical arguments and the results of animal and model circulation experiments.

The purpose of this communication is to describe the indicator dilution curves obtained by injecting blue dye into the left atria of 43 patients with known mitral valvular lesions, and to consider their interpretation in clinical practice.

\section{MethodS AND SubJeCtS}

Forty-three patients referred for diagnostic cardiac catheterization were selected for this study provided they were free from evidence of ischæmic, hypertensive, or aortic valvular disease, and from aneurysmal dilatation of the left atrium (vide infra). None had congestive heart failure within three months of investigation, and each was examined clinically, cardiographically, and radiologically. Three patients were considered to have slight mitral stenosis and six slight mitral incompetence. Their symptoms were mild, and pulmonary arterial systolic pressures measured less than $25 \mathrm{~mm}$. Hg.

Thirty-four patients were severely disabled, and Mr. G. H. Wooler examined the mitral valve at valvotomy or open heart operation. He found stenosis without regurgitation in 9 , and some degree of regurgitation in 25 . Of the 25 with regurgitation the long diameter of the mitral orifice measured $1.5 \mathrm{~cm}$. or less in $9 ; 1.6-2.9 \mathrm{~cm}$. in 9 ; and $3.0 \mathrm{~cm}$. or more in 7 . Patients with orifices of $1.5 \mathrm{~cm}$. or less have clinical and hæmodynamic signs of obstruction, which distinguish them from those with larger orifices (Nixon and Wooler, 1960; Nixon and Wooler, 1961), and patients with orifices of $3.0 \mathrm{~cm}$. or more may be regarded as disabled from regurgitation without obstruction.

Right and transeptal left heart catheterization was performed in the manner described by Nixon 
(1960). Knowing that small differences in systemic vascular resistance may cause large changes in mitral regurgitation, great care was taken by training patient and staff and by using sedation to produce a basal steady state. Records were not used in this study if the patient's discomfort or changes in atrial and arterial pressure or in oxygen consumption indicated departure from the steady state during catheterization. Cardiac output was measured by the Fick principle at the time of the right heart injection of blue dye.

Indicator dilution curves were obtained by injecting blue dye into the right ventricular outflow tract or pulmonary artery, and into the left atrium within $2.0 \mathrm{~cm}$. of the fossa ovalis (Fig. 1-6).

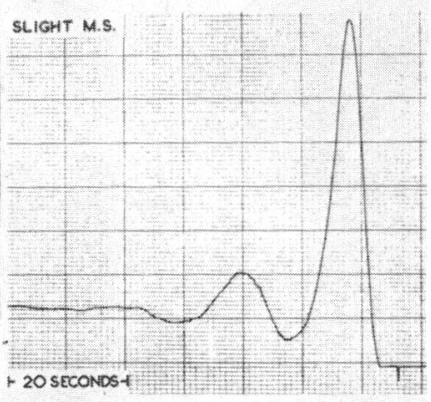

Fig. 1.-Ear-piece dilution curve in slight mitral stenosis. Left atrial injection.

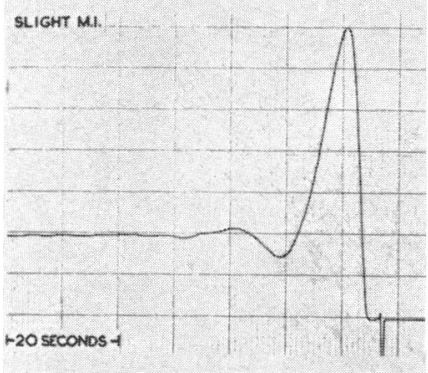

FIG. 2.-Ear-piece dilution curve in slight mitral regurgitation. Left atrial injection.

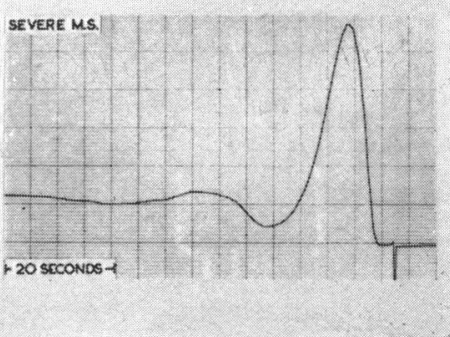

FIG. 3.-Ear-piece dilution curve in severe mitral stenosis. Left atrial injection.

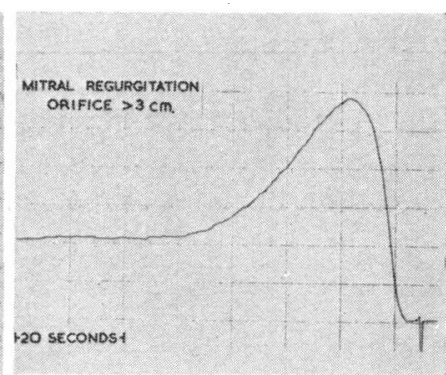

Fig.6.-Ear-piece dilution curve in severe mitral regurgitation. Left atrial injection. from a patient with curve mitral obstruction and slight regurgitation. Left atrial injection.

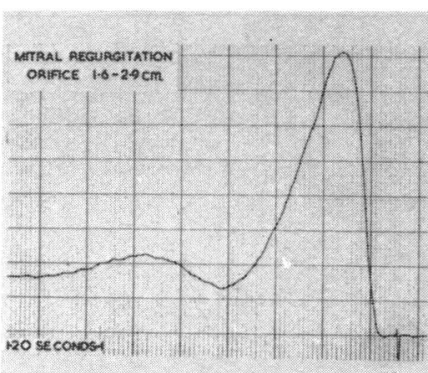

FIG. 5.-Ear-piece dilution curve from a patient severely disabled by mitral stenosis and regurgitation. Left atrial injection.

The curves were recorded with the Cambridge ear-piece and dye dilution apparatus. In the 0 past ear-piece curves have been considered grossly slurred, and therefore unsuitable for assessing valvular incompetence (Wood and Woodward, 1957; Hancock, 1959). The slurring is most easily detected in loss of the dip of least concentration between initial and recirculation peaks (Fig. 7). An arterial cuvette curve free from slurring shows a dip: recirculation peak ratio of 6:10 or less when indicator is injected into the pulmonary artery of normal subjects (Carter, Swan, and Wood 1959). The Cambridge ear-piece was tested for slurring in this laboratory by measuring this ratio in 43 curves obtained from pulmonary arterial or left atrial injection of blue 
dye in patients free from valvular incompetence and shunts, but not necessarıy trom heart enlargement. The results were as follows.

Pulmonary arterial injection: average value $0 \cdot 42$ (range $0 \cdot 7-0 \cdot 3$ ).

Left atrial injection: average value 0.36 (range $0 \cdot 46-0 \cdot 2$ ).

It was considered that these results indicated sufficient freedom from slurring to justify use of the Cambridge ear-piece in a study of valvular incompetence (Fig. 1-6).

The Cambridge ear-piece is simpler to use than a cuvette, is light in weight, has small photoelectric cells, and can be tested easily. The amplifier-recording system requires $1.5 \mathrm{sec}$. to show 90 per cent of fullscale deflection. The apparatus has certain advantages. 1. Pulsations are eliminated by opposing signals from red and infra-red cells without the application of damping. 2. Alterations in response caused by accumulation of dye from repeated injection (Lambert-Beer's law) can be corrected by increasing sensitivity to such a point that each curve is written with the same effective sensitivity. 3. Slow drift from temperature change is eliminated by a capacitor introduced into the infrared cell circuit.

The dose of Coomassie blue dye (Taylor and Thorp, 1959) for each curve was $0.8-1.0 \mathrm{mg}$. $/ \mathrm{kg}$. body weight. Dye was injected into the left atrium through $90 \mathrm{~mm}$. of P.E. 50 catheter during a period of 2 seconds. The curves were calibrated by measuring the "tail height" in the second minute after injection and determining the concentration of dye in a sample of blood withdrawn during the same period (Taylor and Shillingford, 1959).

Left Atrial Size. Left atrial enlargement distorts indicator dilution curves and must be taken into account in the assessment of mitral regurgitation. Measurement of left atrial volume by biplane angiocardiography was considered unjustifiable. The patients were ranked according to the area of the left atrial shadow as shown on 6-ft. penetrating postero-anterior chest radiographs and measured by planimetry. Patients with atrial areas measuring more than $100 \mathrm{~cm}^{2}$ were excluded from the present study because such atrial enlargement can cause gross dye curve abnormality irrespective of whether stenosis or incompetence is the underlying mitral valvular lesion (Fig. 8).
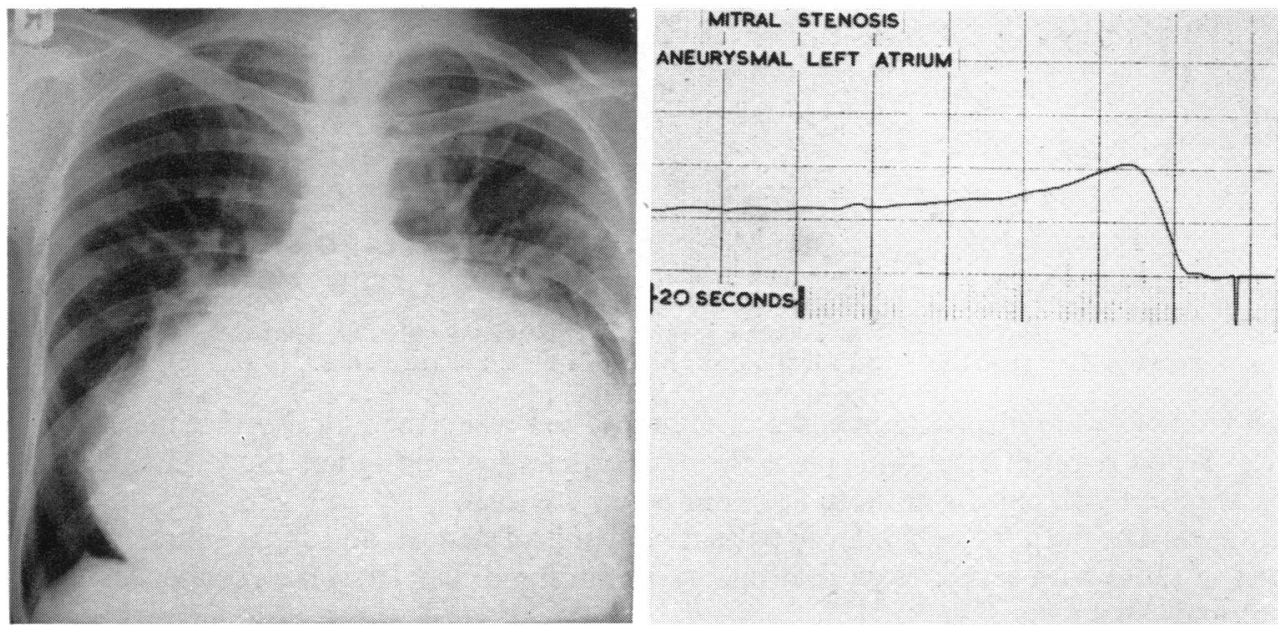

FIG. 8.-Chest radiograph and ear-piece dilution curve (left atrial injection) from a patient with severe mitral stenosis and aneurysmal dilatation of the left atrium. 
Calculations from Dye Curves. Spread/appearance time ratio (Shillingford, 1958) was calculated from curves obtained by right ventricular or pulmonary arterial injection of dye, because measurement of appearance time from left heart injection was considered insufficiently accurate and liable to variation with the phase of the cardiac cycle.

Cardiac output, mean circulation time, Hamilton's volume, and factor "X" (Hamilton et al., 1932), and Newman's volume (Newman et al., 1951; Meier and Zierler, 1954) were calculated, from curves obtained by left atrial injection in the manner described in the Appendix.*

The ratio of least concentration between primary and recirculation peaks to recirculation peak concentration (C.L./C.R.-Wood and Woodward, 1957) was obtained from uncalibrated curves obtained by left atrial injection of dye (Fig. 7).

\section{RESULTS}

Left Atrial Area. Atria were larger in mitral regurgitation than in mitral stenosis, whether the disease was slight or severe (Fig. 9). When the mitral orifice measured $1.5 \mathrm{~cm}$. or less regurgitation patients ranked with those suffering from severe stenosis. It made no difference to atrial size whether the incompetent orifice measured $1.6-2.9 \mathrm{~cm}$. or more than $3.0 \mathrm{~cm}$.

Volume and Cardiac Output. The results are given in Table I. The average Hamilton and

\section{left atrial area}

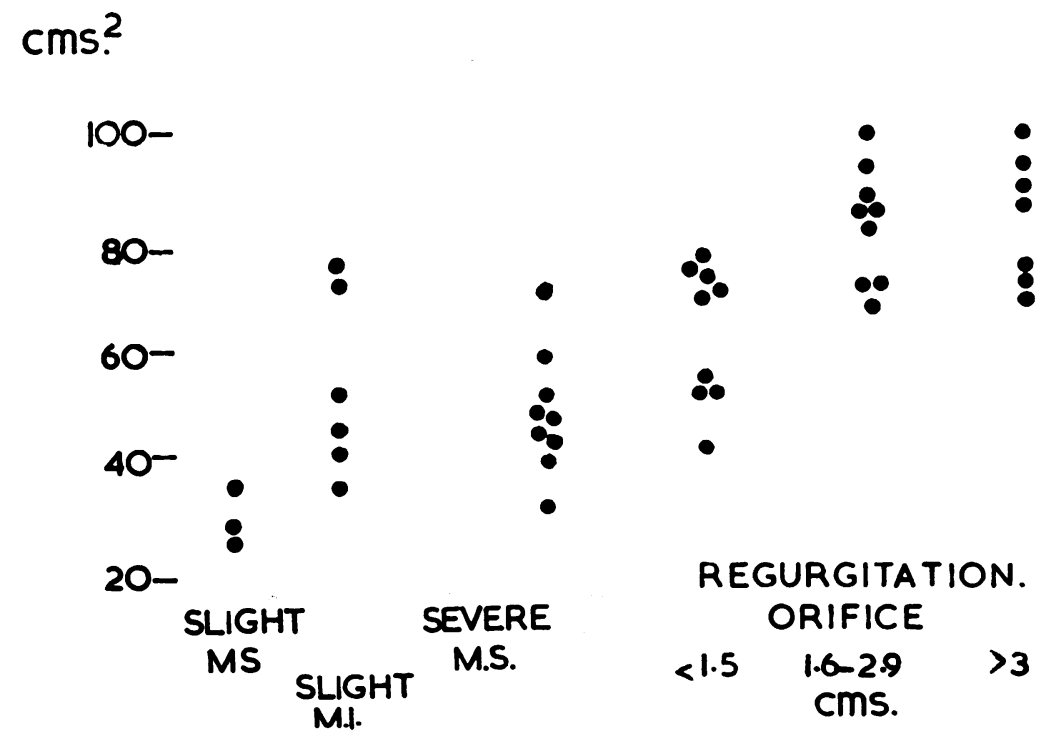

Fig. 9.-Left atrial area in 43 cases of mitral valvular disease. M.S. = mitral stenosis; M.I. = mitral incompetence.

Newman volumes were increased in cases of regurgitation when the mitral orifice measured $1.5 \mathrm{~cm}$. or more. When regurgitation took place through orifices measuring less than $1.5 \mathrm{~cm}$. the average volumes were not different from those found in severe stenosis.

Average cardiac output was similar in patients with mitral stenosis and slight mitral regurgitation, and lower in those with severe regurgitation. This finding differs from the experience of Levinson, Carleton, and Abelmann (1959).

Mean Circulation Time (M.C.T.). Much higher values were obtained in severe mitral 
TABLE I

Volume Between Injection and Sampling Site Correlated with the State of Mitral Valve and Cardiac OUTPUT

(Figures indicating range of results are shown in parenthesis)

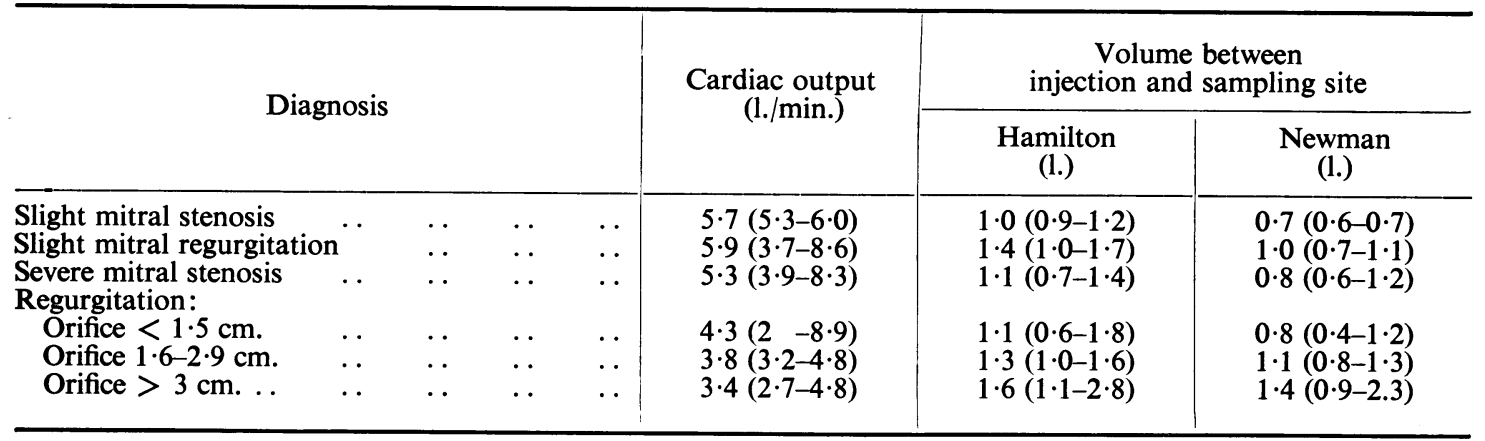

* Copies of the Appendix may be obtained from the authors.

mean

\section{circulation \\ time.}

\section{seconds}

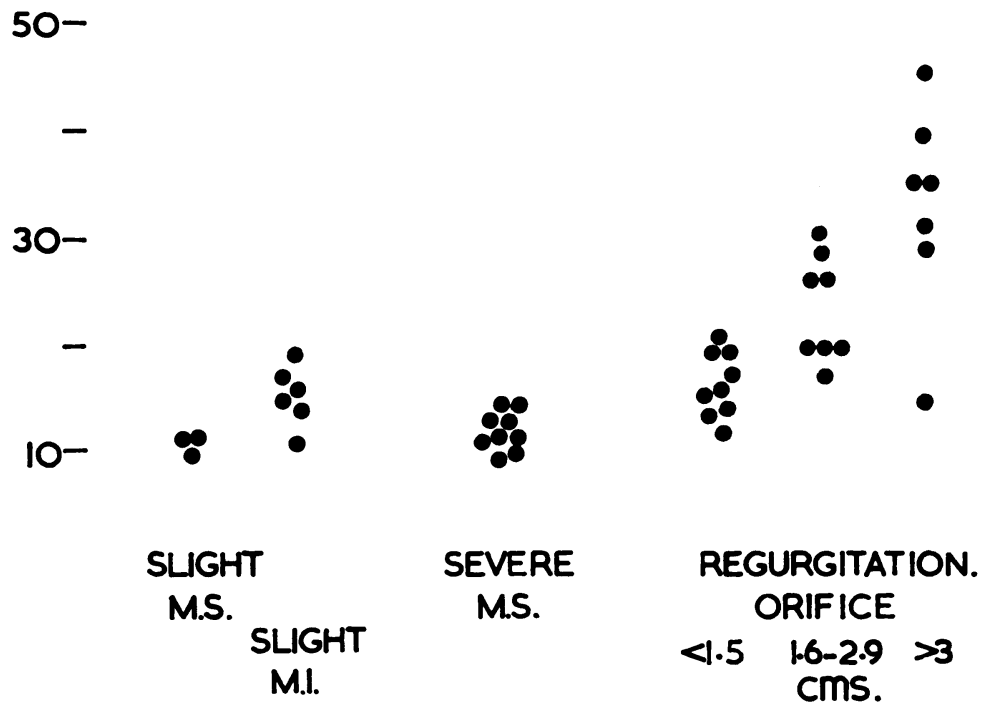

FIG. 10.-Mean circulation time in 43 cases of mitral valvular disease, calculated from ear-piece curves following left atrial injection.

regurgitation than in severe mitral stenosis (Fig. 10). Patients with regurgitation ranked with the severely stenotic when their orifices measured $1.5 \mathrm{~cm}$. or less.

The prolongation of M.C.T. resulted from decreased cardiac output as well as from increased Hamilton volume. The observation that it does not reflect enlarged volume alone differs from the conclusion of Levinson et al. (1959).

Ratio of least concentration between primary and recirculation peaks to concentration at recirculation peak (C.L./C.R.) separated patients with slight mitral regurgitation from those with slight or severe stenosis. In mitral regurgitation it distinguished patients with orifices measuring $1.5 \mathrm{~cm}$. 
or less from those with orifices larger than $3.0 \mathrm{~cm}$. Patients with regurgitation through small orifices tended to rank with the severely stenotic (Fig. 11).

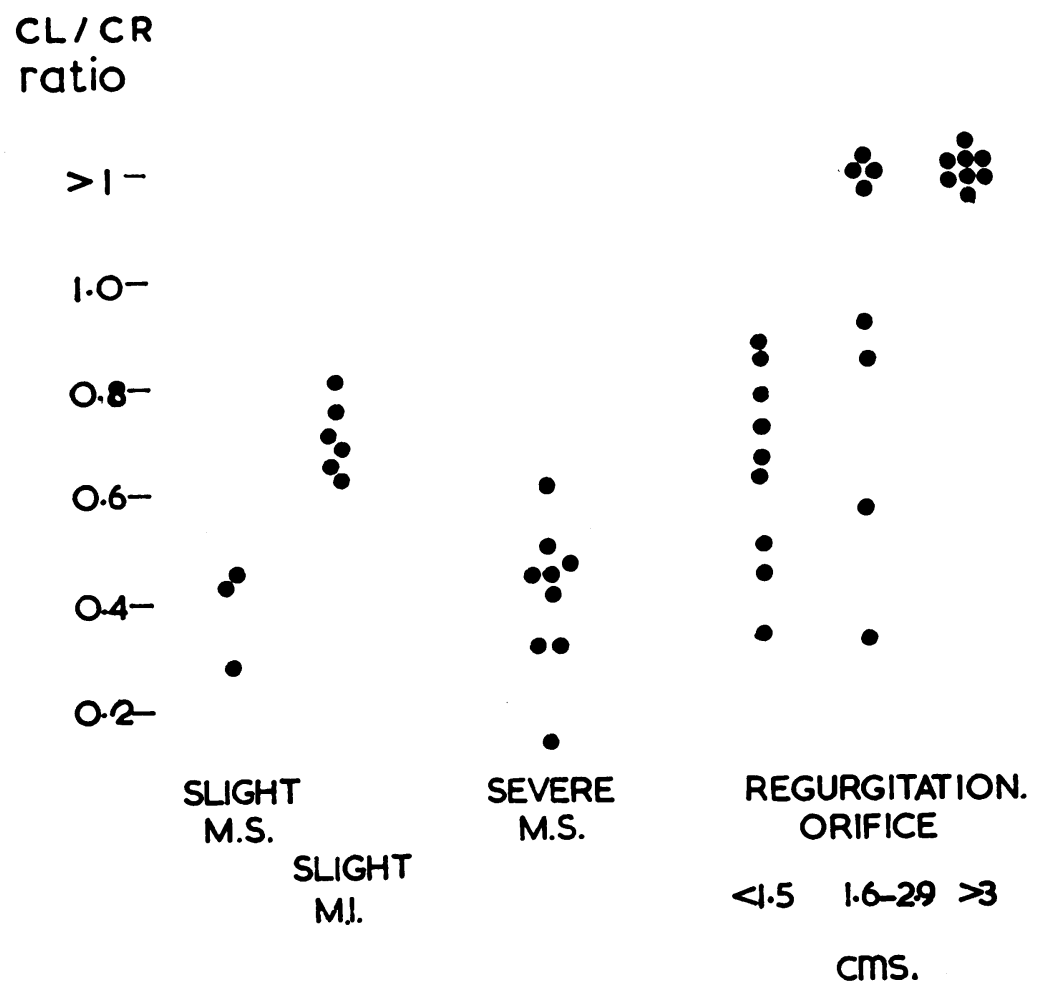

Fig. 11.-Ratio of least concentration to recirculation peak concentration in 43 cases of mitral valvular disease, calculated from ear-piece curves following left atrial injection.

Spread/Appearance Time (Fig. 12). With one exception this formula separated cases of regurgitation with large orifices from cases with small orifices, and from the severely stenotic.

Hamilton's Factor " $X$ " (Fig. 13). Patients with regurgitation through small orifices ranked with the severely stenotic and were distinguished from those with large orifices.

Given the observed range of left atrial size and cardiac output in severely disabled patients with $\bigcirc$ mitral regurgitation it is evident that C.L./C.R. distinguishes cases with orifices greater than $3.0 \mathrm{~cm}$. from those with orifices measuring $1.5 \mathrm{~cm}$. or less and separates patients with slight mitral regurgitation from those with stenosis. It is noteworthy that the very ratio for which ear-piece o curves have been considered unsuitable (Wood and Woodward, 1957; Hancock, 1959) is their most ${ }^{\circ}$ effective tool. Spread/appearance time and factor $\mathbf{X}$ are less efficient.

\section{Discussion \\ Mechanism of Distortion of the Dilution Curve of Valvular Incompetence}

In health the left ventricle ejects its stroke volume and retains a residual volume that may amount 0 to 46 per cent of the end-diastolic volume (Holt, 1956). In mitral regurgitation the left ventricle $\frac{0}{\mathbb{O}}$ and atrium must enlarge to accommodate the regurgitant volume if the cardiac output is to be maintained without great increase in the heart rate. The extent of the enlargement may not be related $\stackrel{\mathscr{Q}}{Q}$ 


\section{spread/appearance time ratio}

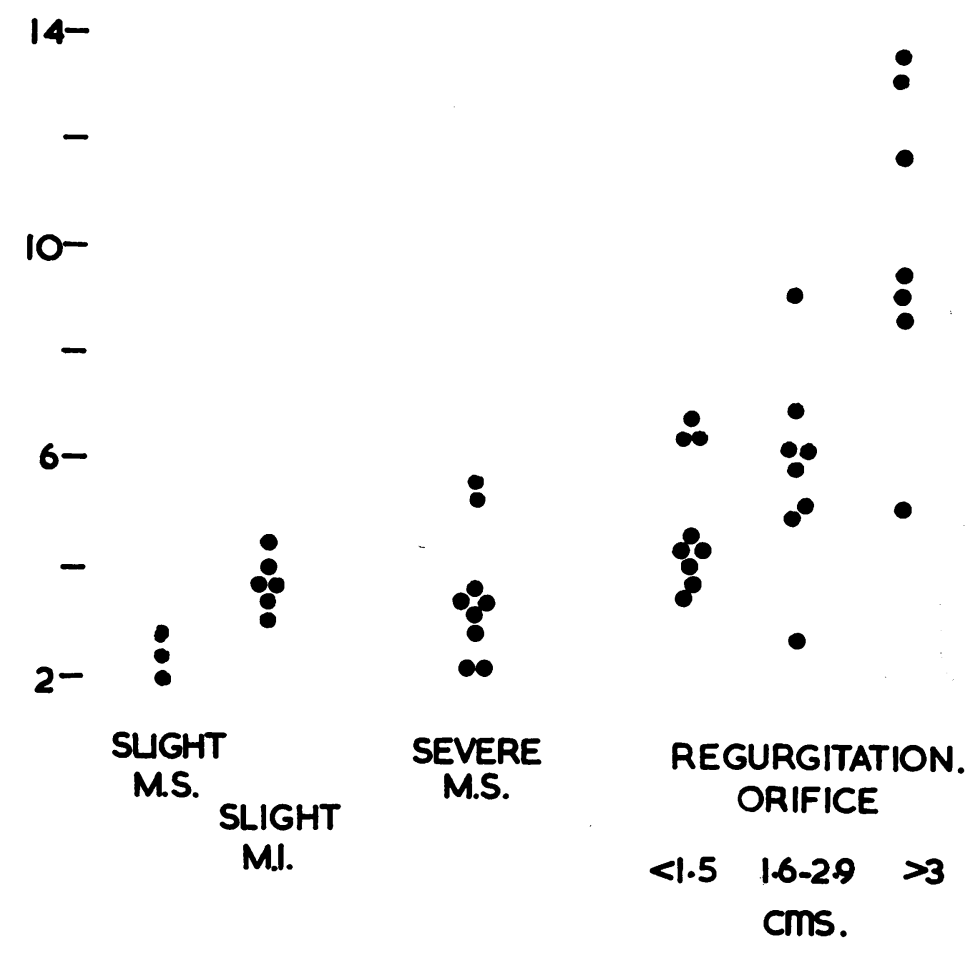

FIG. 12.-Ratio of spread to appearance time in 43 cases of mitral valvular disease, calculated from ear-piece curves following pulmonary arterial or right ventricular injection.

directly or closely to the volume of the regurgitant flow, and yet it has great influence upon the shape of the dilution curve (Conn, 1959). The size of the left atrium is particularly important because enlargement of atrial residual volume increases the amount of blood exposed to mixing with the indicator regurgitated from the ventricle and correspondingly increases the distortion of the dilution curve (Levinson et al., 1959). If the residual volume is small the regurgitant volume must be improbably great to impose a slow downslope upon the dilution curve (Emanuel, Lacy, and Newman 1959). The relative roles of regurgitant and residual volume abnormality in producing dilutioncurve distortion in valvular incompetence may be illustrated by contrasting the curves recorded in severe mitral regurgitation, where there is atrial enlargement (Fig. 6), with the curve obtained in a similar manner from a patient with severe aortic regurgitation in whom the blood pressure is $180 / 40 \mathrm{~mm}$. Hg (Fig. 14). The downslope of this curve is normal, and it is probable that the blood regurgitated into the ventricle during diastole was completely ejected during the next systole and not subjected to excessive mixing with an increased residual volume.

In mitral regurgitation the abnormalities of stroke volume, residual volume, and mixing (Phinney, Cotton, and Shillingford, 1961), and the presence of a regurgitant volume, distort the dilution curve in a characteristic manner. The gradient of the downslope is reduced (Korner and Shillingford, 1955); the peak concentration is reduced, the time components are prolonged, and the recirculation peak may disappear (Woodward, Burchell, and Wood, 1957). 


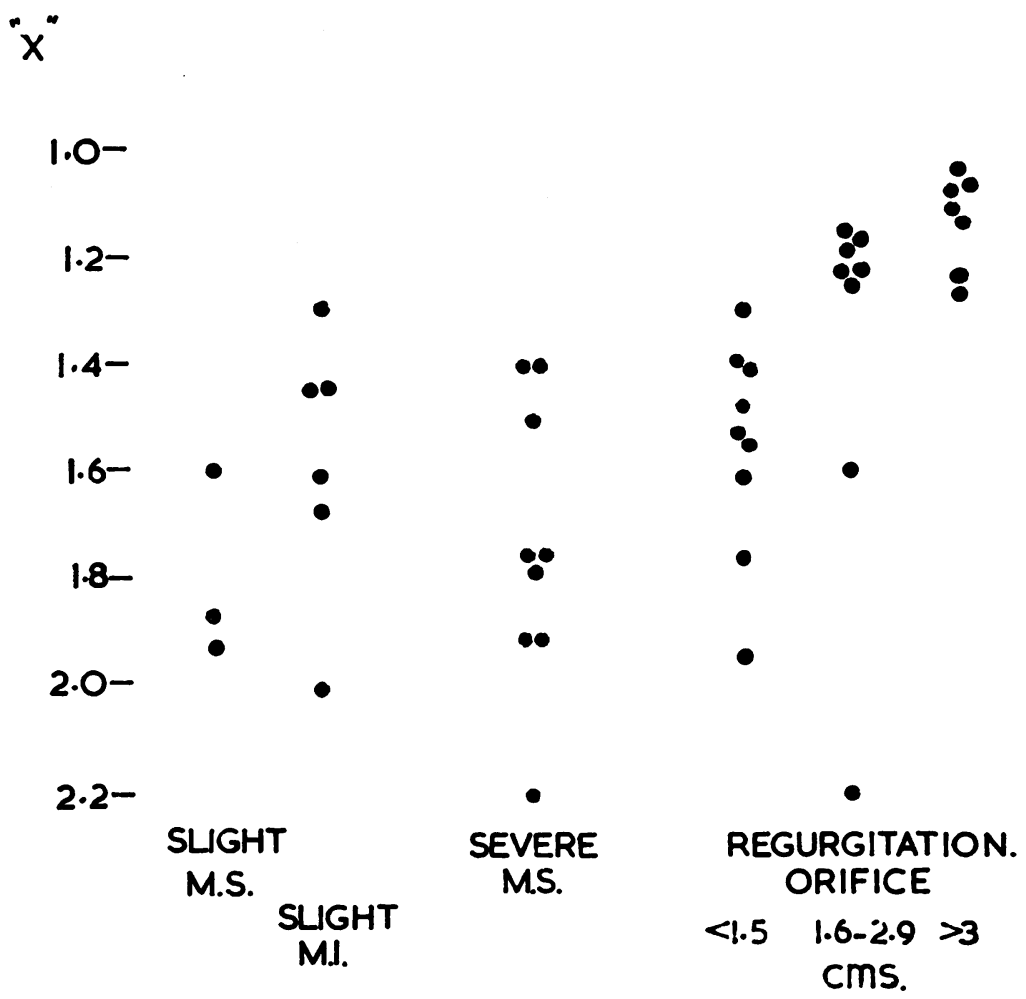

Fig. 13. - Hamilton's factor " $X$ " in 43 cases of mitral valvular disease, calculated from ear-piece curves following left atrial injection.
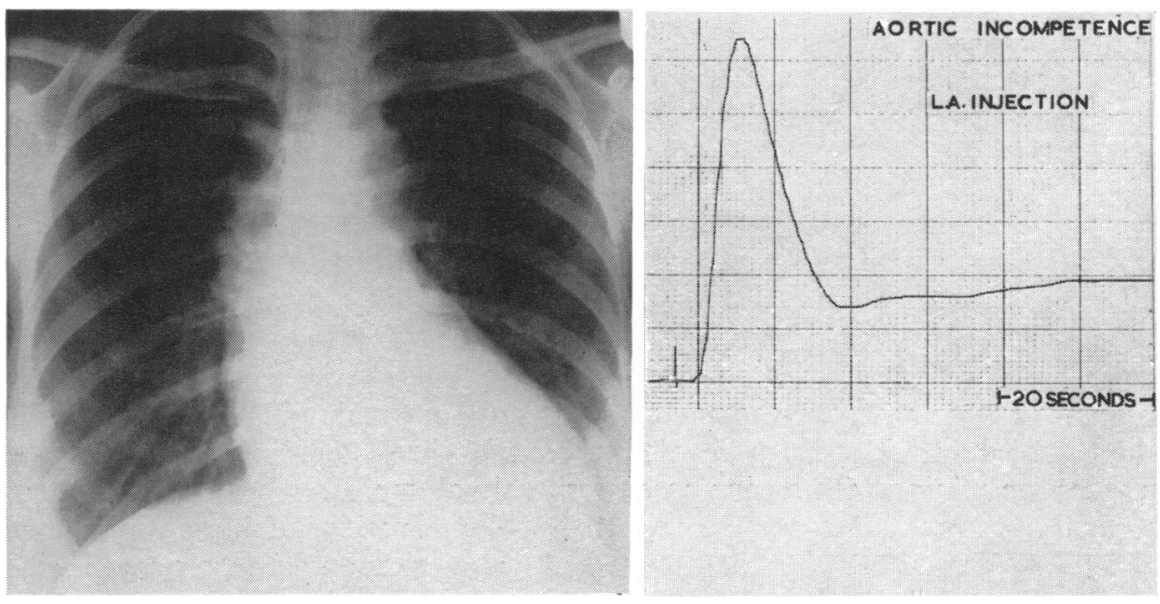

FIG. 14.-Chest radiograph and ear-piece dilution curve in severe aortic valvular regurgitation (left atrial injection). 


\section{The Measurement of Regurgitation}

Theoretically the indicator dilution technique is the method most likely to succeed in measuring the volume and mixing disorders of valvular incompetence, but in practice serious obstacles are present. The first and foremost is the failure of dye to mix instantaneously and homogeneously with all the blood in the chamber into which it is injected (Hamilton et al., 1932). When dye is injected rapidly the curve may vary according to the phase of the cardiac cycle at the moment of injection (Meier and Zierler, 1954): for example, indicator injected into the atrium in late diastole may be ejected preferentially from the ventricle and scarcely mix with the ventricular residual volume (Swan and Beck, 1960). The accuracy of measurement may be affected by atrial size and elasticity (Hoffman and Rowe, 1959; Thorburn et al., 1959; Levinson et al., 1959), and by the distances between injection and sampling sites and the incompetent valve (Emanuel et al., 1959; Korner et al., 1959). It is necessary to assume that the sampling artery receives representative blood flow, for if it receives richly or poorly dyed non-representative samples, neither its blood nor the blood from any other artery can give a valid dilution curve (Dow, 1956). It is necessary to assume that all the injected dye is accounted for in the curve of primary circulation, but a fraction of indicator following a slow pulmonary pathway may not be counted in the curve of primary circulation because it fails to reach the sampling site before the recirculating indicator, and dye moving fast may be counted twice in the primary curve (Dow, 1956). A large error is introduced when insufficient downstroke exists for the point of recirculation to be clearly recognized (Meier and Zierler, 1954). It may not be valid to assume that the effects of recirculating indicator can be excluded from semi-logarithmic plots in every case (Dow, 1956), and the limitation of graphic methods of plotting dilution curves may cause error of measurement of regurgitant volume (McClure et al., 1959). Technical problems are numerous. For example, the use of a catheter for sampling may affect the shape of the dilution curve sufficiently to preclude accurate measurement of regurgitant volume in vivo (McClure et al., 1959; Sheppard, Jones, and Couch, 1959; Sherman, et al., 1959), and commercial instruments that record dilution curves continuously by photometry may be too inaccurate for the measurement of cardiac volumes (Dow, 1956).

Multiple sampling techniques that involve injection into the left ventricle and sampling from the left atrium and the aorta do not escape the requirements of instantaneous and homologous mixing, representative sampling, and accurate recording that apply to single curves. Factors responsible 'for the inaccuracy of their estimates of regurgitation have been examined by Levinson et al. (1960).

Attempts have been made to extrapolate the measurements of regurgitant flow from dog and model circulation experiments to clinical valvular regurgitation. In dogs thoracotomy may have great effect on cardiac volumes (Rushmer et al., 1956), and the acute experiment may fail to reproduce the conditions found in chronic rheumatic heart disease. In many model experiments forward flow is maintained by increasing heart rate with regurgitation, and there is failure to allow for the volume changes that are the obligatory concomitants of clinical valvular regurgitation. Dow (1956) remarked that "hydraulic models, in general, demonstrate only how close one can build to theoretical assumptions, not at all how close the theory or the model approximates to the cardiovascular system."

From the foregoing discussion it is clear that measurement of regurgitant volume in the face of independently variable changes in residual volume, stroke volume, and mixing is a task sufficiently formidable to justify an empirical approach to the interpretation of dilution curves in clinical mitral regurgitation. It is reasonable to assess the diagnostic value of a simple and constant dye dilution technique even though it may be impossible to measure the factors that influence the result. But in order to assess the diagnostic value of a dye technique it is necessary to select formulæ that express distortion of the dilution curve, and to choose independent estimates of valvular incompetence for comparison.

Some formulæ that describe the dilution curve are employed only to express the degree of distortion found in time components, downslope, and recirculation peak. Others are based upon the 
assumption that mixing is closely related to regurgitation, and are used in attempts to isolate the effects of increased mixing from the results of enlarged cardiac volume and reduced cardiac output.

\section{Formula Expressing Dilution Curve Distortion}

Appearance time (A.T.) is the interval between the injection of indicator and its appearance at the sampling point. It shortens with regurgitation (Shillingford, 1958) and lengthens with increases in volume. In clinical regurgitation the effects may cancel out, or either may predominate (Levinson et al., 1959).

Mean circulation time (M.C.T.) is the mean time required for dye to travel from the injection to the sampling site. It is unaffected by regurgitation per se (Shillingford, 1958), but it is increased in clinical regurgitation as a result of enlargement of central volume and reduction in cardiac output.

Build-up time (B.T.) is the interval between the appearance of dye at the sampling point and the peak of the primary curve. It is affected by changes in regurgitation, flow, and volume in a similar manner to the appearance time.

Flattening of the downslope of the curve of primary circulation has been described by a number of methods. They include disappearance time (D.T.), slope, reciprocal of the slope, 10-second disappearance ratio (Levinson et al., 1959), and the time required for a tenfold decrease in concentration (Hancock, 1959). All indicate flattening from increased volume and mixing, and from decreased forward flow, and should, if normal ranges were known, provide a guide to the total abnormalities of clinical regurgitation.

The ratio of least concentration between primary and recirculation peaks to recirculation peak concentration (C.L./C.R.; Wood and Woodward, 1957), like the downslope of primary circulation, is affected by changes in mixing, volume, and cardiac output. It is also affected by incompetence of valves not situated between injection and sampling sites, and by changes in whole body blood volume.

\section{Formula to Isolate the Effects of Increased Mixing from the Results of Increased Volume and Decreased Cardiac Output}

D.T./B.T. (Woodward et al., 1957). The ratio of disappearance time to build-up time enhances the results of increased mixing from regurgitation and tends to cancel out the volume and flow effects.

D.T./M.C.T. (Levinson et al., 1959). The ratio of disappearance time to mean circulation time does not enhance the result of mixing from regurgitation so much as D.T./B.T., but it also tends to cancel out flow and volume effects.

Disappearance Ratio (Fox and Wood, 1957). This is calculated by dividing concentration at the primary circulation peak into concentration at a time equal to peak concentration time plus build up time. The effects of volume, flow, and mixing upon build up time, peak concentration, and downslope enter into the calculation which enhances the result of mixing at the expense of volume and flow effects.

Spread/Appearance Time Ratio (Shillingford, 1958). Spread is proportional to mixing and volume and inversely proportional to flow; and appearance time is proportional to volume and inversely : proportional to flow and mixing. Theoretically the use of the ratio should tend to cancel the effects of flow and volume, and to enhance the result of increased mixing. In clinical mitral regurgitation the formula may be less successful because appearance time is frequently prolonged (Levinson et al., 1959).

The Reciprocal of the Slope and the Variance of the Dilution Curve (Korner and Shillingford, 1955, 1956). If the dilution curve is treated as a frequency distribution of dye particles, the effect of regur- 0 gitation may be regarded as a diminished probability of forward movement of a given dye particle. The probability of forward movement is assessed from the downslope and the variance, which are $\mathbb{D}$ expressions of dispersion. Calculation of regurgitant flow depends upon accurate measurement of $\frac{\mathrm{Q}}{\alpha}$ 
cardiac output and intravascular volumes for the formulation of appropriate regression equations. The constants in the regression equations (see Appendix) are closely related to Hamilton's factor " $\mathrm{X}$ " employed in the present study, and similarly are expressions of biological departure from the imaginary model chamber. It may be seen that factor " $X$ " varies greatly from patient to patient (Fig. 13). Its use in the assessment of clinical mitral regurgitation has not previously been reported.

"Residual volume" calculated from Newman's formula.

Slope $=$ Heart rate $\times \log _{\mathrm{e}} \frac{\text { Residual Volume }}{\text { Residual Volume }+ \text { Stroke Volume }}$

This is an hypothetical quantity that includes regurgitant volume with true residual volume, and increases in clinical mitral regurgitation. To assess the severity of regurgitation Conn (1959) related it to Hamilton volume, and Levinson et al. (1959) related it to stroke volume.

From all the possible methods of expressing dilution curve distortion, mean circulation time, C.L./C.R., spread/appearance time, and Hamilton's factor " $X$ " were selected as sufficiently representative for this study.

\section{Independent Estimates of Valvular Regurgitation}

It is not easy to create yard-sticks for the assessment of dilution curves in mitral regurgitation. Clinical signs are particularly liable to misinterpretation since left ventricular enlargement and loud systolic murmurs may occur with regurgitation through small obstructed mitral orifices (Nixon and Wooler, 1960); the absence of mitral opening and closing snaps may indicate the rigidity of the aortic leaflet rather than the severity of regurgitation (Nixon, Wooler, and Radigan, 1960); and cases of severe mitral regurgitation frequently have loud mitral diastolic murmurs that begin at the left atrial annular ascent point (Nixon and Wooler, 1960; Nixon, 1961a, b).

In the case of severely disabled patients it is reasonable to assume that small mitral orifices cause severe obstruction and permit little regurgitation, and that larger orifices permit greater regurgitation with less obstruction. Consequently it is reasonable to rank severely disabled cases of mitral regurgitation according to the size of the mitral orifice. It is easier to measure the long diameter of the orifice (Goodwin et al., 1955) than to estimate its area (McDonald et al., 1957).

This study has shown that the ratio C.L./C.R. allows patients with slight regurgitation to be distinguished from those with slight stenosis, and disabled patients with large mitral orifices from those with small orifices. The ratio reflects all the mixing and volume abnormalities of valvular incompetence without attempting to isolate the effects of one from its concomitants. Calculation of the ratio does not require the dilution curve to be replotted onto semilogarithmic paper, nor does it require regression equations. The ease and speed with which the calculation is made during cardiac catheterization is advantageous in clinical practice. Bercu et al. (1958) and Levinson et al. (1959) calculated C.L./C.R. from cuvette curves and commented favourably upon its value. Since then the introduction of an ear-piece that produces curves sufficiently free from slurring and obviates the need for catheter-cuvette sampling systems has greatly simplified the clinical use of the ratio.

If the yard-stick of mitral orifice size is accepted, it is apparent that indicator dilution curves are not sensitive indicators of valvular incompetence, and the ranking of patients that is achieved probably depends upon the changes in cardiac output, volume, and mixing being roughly proportional to the severity of the mitral regurgitation. Failure to rank cases of regurgitation with the ratio C.L./C.R. may be anticipated if cardiac output and volumes are unusually great or small. It is likely that batteries of complicated indices will be misled by these exceptions; and it is to be doubted whether the use of a cuvette would greatly improve the situation. Accurate measurement of regurgitant flow must await sampling without catheter, and the solution of the problems of mixing and representative sampling.

\section{SUMMARY}

Coomassie blue dye was injected consecutively into the right heart and left atrium of 43 cases of mitral valvular disease free from gross left atrial enlargement. Unslurred ear-piece dilution 
curves were examined by methods that merely expressed distortion, and by methods that dis- $\stackrel{\vec{C}}{.}$ criminated between the effects of regurgitation and the results of decreased cardiac output and $\overrightarrow{\vec{F}^{*}}$ increased heart volume. The severity of regurgitation was independently assessed from the size $\frac{7}{\sigma}$ of the mitral orifice in severely disabled cases.

The ratio of least concentration to recirculation peak concentration is the least discriminative of the indices tested, and yet it was the most successful in ranking patients according to the severity of regurgitation. The reason may be that it is affected by all the circulatory abnormalities of clinical mitral regurgitation.

Biological and technical impediments to the accurate measurement of regurgitant flow and to the clinical assessment of indicator dilution curves are discussed.

An appendix, which may be obtained from the authors, offers a simple method for measuring areas under dilution curves, and analyses the theoretical relation between dilution curve downstroke, flow, and volume. It describes the relationships between the Korner-Shillingford regression equations and the arguments of Hamilton and Newman.

We are deeply indebted to Mr. G. H. Wooler and Professor R. E. Tunbridge for making this work possible, and for $\bigcirc$ their support and encouragement. Staff Nurse E. M. Kirkbride, Mrs. J. Blake, Miss V. Tuke, Mr. G. Naylor and $\rightarrow$ Mrs. L. Bray are to be thanked for their assistance. The Board of Governors of the United Leeds Hospitals, the $\Theta$ Medical Research Council, and private benefactors have generously provided funds. The salary of one of us $\$$ (H.M.S.) is being paid by the Trustees of the Nuffield Foundation.

\section{REFERENCES}

Bercu, B. A., Diettert, G. A., Pund, E. E., and Danforth, W. H. (1958). Circulation, 18, 694. Carter, S. A., Swan, H. J. C., and Wood, E. H. (1959). Circulation, 19, 430.

Conn, H. L. (1959). Progr. cardiovasc. Dis., 2, 166.

Dow, P. (1956). Physiol. Rev., 36, 77.

Emanuel, R. W., Lacy, W. W., and Newman, E. V. (1959). Circulat. Res., 7, 141.

Fox, I. J., and Wood, E. H. (1957). J. Lab. clin. Med., 50, 598.

Goodwin, J. F., Hunter, J. D., Cleland, W. P., Davies, L. G., and Steiner, R. E. (1955). Brit. med. J., 2, 573.

Hancock, E. W. (1959). Guy's Hosp. Rep., 108, 349.

Hamilton, W. F., Moore, J. W., Kinsman, J. M., and Spurling, R. G. (1932). Amer. J. Physiol., 99, 534.

Hoffman, J. I. E., and Rowe, G. G. (1959). J. clin. Invest., 38, 146.

Holt, J. P. (1956). Circulat. Res., 4, 187.

Korner, P. I., and Shillingford, J.' P. (1955). Clin. Sci., 14, 553.

二, Thorburn, G. D., and Edwards, A. W. T. (1959). Clin. Sci., 18, 321.

Levinson, G. E., Carleton, R. A., and Abelmann, W. H. (1959). Amer. Heart J., 58, 873.

Levinson, Katznelson, G., Stein, S. W., and Abelmann, W. H. (1960). Circulation, 22, 778.

McDonald, L., Dealy, J. B., Rabinowitz, M., and Dexter, L. (1957). Medicine (Baltimore), 36, 237.

McClure, J. A., Lacy, W. W., Latimer, P., and Newman, E. V. (1959). Circulat. Res., 7, 794.

Meier, P., and Zierler, K. L. (1954). J. appl. Physiol., 6, 731.

Newman, E. V., Merrell, M., Genecin, A., Monge, C., Milnor, W. R., and McKeever, W. P. (1951). Circulation, 4, 735.

Nixon, P. G. F. (1960). Thorax, 15, 225.

(1961a). Brit. Heart J., 23, 677.

(1961b). Brit. Heart J., 23, 462 .

二, and Wooler, G. H. (1960). Brit. med. J., $2,1122$.

二, and (1961). Brit. Heart J., 23, 161.

$\longrightarrow$, and Radigan, L. R. (1960). Brit. Heart J., 22, 395.

Phinney, A. O., Cotton, K., and Shillingford, J. P. (1961). Clin. Sci., $20,75$.

Rushmer, R. F., Finlayson, B. L., and Nash, A. A. (1956). Circulat. Res., 4, 337.

Sheppard, C. W., Jones, M. P., and Couch, B. L. (1959). Circulat. Res., 7, 895.

Sherman, H., Schlant, R. C., Kraus, W. L., and Moore, C. B. (1959). Circulat. Res., 7, 303.

Shillingford, J. P. (1958). Brit. Heart J., 20, 229.

Swan, H. J. C., and Beck, W. (1960). Circulat. Res., 8, 5.

Taylor, S. H., and Shillingford, J. P. (1959). Brit. Heart J., $21,497$.

Taylo, and Thorp, J. M. (1959). Brit. Heart J., 21, 492.

Thorburn, G. D., Korner, P. I., and Stephens, J. (1959). Clin. Sci., 18, 345.

Wood, E. H., and Woodward, E. (1957). Proc. Mayo Clin., 32, 536.

Woodward, E., Burchell, H. B., and Wood, E. H. (1957). Proc. Mayo Clinic, 32, 518. 